\title{
Extended phage locus typing of Salmonella enterica serovar Typhimurium, using multiplex PCR-based reverse line blot hybridization
}

Correspondence

Gwendolyn L. Gilbert

l.gilbert@usyd.edu.au

Received 15 November 2007

Accepted 15 February 2008

\author{
Qinning Wang, Fanrong Kong, Peter Jelfs and Gwendolyn L. Gilbert
}

Centre for Infectious Diseases and Microbiology - Public Health, Institute of Clinical Pathology and Medical Research (ICPMR), Westmead, New South Wales 2145, Australia

\begin{abstract}
Salmonella enterica serovar Typhimurium ( $S$. Typhimurium) is the commonest pathogen causing food-borne disease among humans and animals in Australia. A multiplex PCR-based reverse line blot (mPCR/RLB) system was developed to rapidly identify $S$. Typhimurium phage types and strains within them. The system comprised 32 biotin-labelled primer sets and 38 amino-labelled probes, based on sequences that were either phage-type-related or derived from temperate phages ST64B, P22, Gifsy-1 or Gifsy-2. The system was developed and evaluated using 168 S. Typhimurium isolates, representing 46 phage types. RLB patterns, based on a combination of positive hybridization and grading of signal intensities, validated by sequencing, differentiated $S$. Typhimurium isolates into 102 types. Some clusters contained isolates belonging to a single phage type while others contained isolates belonging to more than one. Most phage types exhibited at least two RLB profiles. The feasibility of this system was evaluated during investigations of three outbreaks, due to two different phage types. Within each outbreak, isolates showed identical RLB patterns, whereas sporadic isolates of corresponding phage types showed various patterns. The mPCR/RLB system was compared with multilocus variable-number tandem-repeat analysis (MLVA). The two methods demonstrated similar discriminatory abilities. Based on these preliminary results, the mPCR/RLB system is a promising tool for molecular identification of most common $S$. Typhimurium phage types. It could be used as an alternative to, or in conjunction with, MLVA for rapid strain typing during outbreaks.
\end{abstract}

\section{INTRODUCTION}

Salmonella enterica serovar Typhimurium (S. Typhimurium), one of more than 2500 serovars recognized in the genus Salmonella (Popoff et al., 2004), is a major bacterial pathogen that causes food-borne infection (salmonellosis) in humans and animals worldwide. For many years it has been the most common serovar isolated from humans and animals in Australia. In 2006 it accounted for $25.7 \%$ of human and $16.2-30.4 \%$ of chicken and bovine isolates, albeit with considerable geographical and temporal variations (Davos, 2006).

$S$. Typhimurium is a common and diverse serovar that requires further subtyping for surveillance or outbreak investigations. Phage typing (Anderson et al., 1977) is widely used in Australia and Europe to differentiate individual strains of $S$. Typhimurium. However, it is restricted to a few reference laboratories, which causes delays and limits its epidemiological value. Interpretation

Abbreviations: AFLP, amplified fragment length polymorphism; MLVA, multilocus variable-number tandem-repeat analysis; mPCR/RLB, multiplex PCR-based reverse line blot; PT, phage type; RDNC, reaction-doesnot-conform. of results, even by experienced staff, may vary between laboratories. Untypable or 'reaction-does-not-conform' (RDNC) isolates are not uncommon. Often a single phage type (PT) predominates for long periods in a geographical area, making recognition of outbreaks difficult.

Molecular typing methods have been used for further discrimination of Salmonella strains, including PFGE (Guerra et al., 2000), random amplified polymorphic DNA typing (De Cesare et al., 2001), multilocus sequence typing (Fakhr et al., 2005), IS200 typing (Jeoffreys et al., 2001; Soria et al., 1994), ribotyping (Nastasi \& Mammina, 1995), plasmid profiling (Millemann et al., 1995) and amplified fragment length polymorphism (AFLP) analysis (Hu et al., 2002; Lindstedt et al., 2000). Some are relatively difficult to perform, slow and expensive whilst others have limited reproducibility and discriminatory powers. Multilocus variable-number tandem-repeat analysis (MLVA) has been developed for various Salmonella serovars (Cho et al., 2007; Lindstedt et al., 2004; Liu et al., 2003; Witonski et al., 2006). It has allowed a high level of resolution of variant strains of the relatively homogeneous S. Typhimurium definitive type 104 (Lindstedt et al., 2003). 
A number of phage type-specific sequences have been identified in $S$. Typhimurium and its phages and used to distinguish phenotypically related definitive types of $S$. Typhimurium by PCR and sequencing (Hu et al., 2002; Lan et al., 2007; Mikasova et al., 2005; Ross \& Heuzenroeder, 2005). As this involves multiple single PCRs and sequencing, it is time-consuming and unsuitable for routine identification and typing. In this study, we developed a multiplex PCR-based reverse line blot (mPCR/ RLB) assay to rapidly identify most of the phage types currently prevalent in our region and compared the results with those of MLVA.

\section{METHODS}

Bacterial isolates and DNA preparation. One hundred and sixtyeight $S$. Typhimurium isolates, representing 46 phage types, were tested. They had been isolated, mainly from human stool samples, between 2000 and 2005 and referred for serotyping to the New South Wales Enteric Reference Laboratory at the Centre for Infectious Diseases and Microbiology. Phage typing was performed, using the Anderson scheme (Anderson et al., 1977), by the Microbiological Diagnostic Unit, University of Melbourne, or the Australian Salmonella Reference Centre, Institute of Medical and Veterinary Science, Adelaide (Fig. 1).

Isolates from three separate outbreaks were investigated. (i) Outbreak 1: 23 isolates of PT 135 from a suspected outbreak in a drug and alcohol rehabilitation centre in 2004 were compared with two PT 126 isolates and 15 non-outbreak PT 135 isolates (collected between 2001 and 2003). (ii) Outbreak 2: 15 isolates (14 from humans, one from food) of PT 170 from three apparently related restaurant outbreaks in 2006 were compared with 22 sporadic PT 170 isolates (collected between 2001 and 2005). (iii) Outbreak 3: 17 isolates of unknown phage type from an outbreak in November 2006, involving students from a catering college, and six Salmonella isolates of unknown serovar from three types of food were investigated and compared with controls, which were isolates belonging to four common $S$. Typhimurium phage types, namely PT 126, 135, 170 and U290.

All isolates were grown on horse blood agar overnight at $37^{\circ} \mathrm{C}$. Single colonies of each were suspended in $200 \mu \mathrm{l}$ molecular biology grade water and boiled for $10 \mathrm{~min}$. The suspension was centrifuged and the supernatant was used for PCR.

Primers. Thirty-two sets of primers were designed, based on previously published primers and $S$. Typhimurium phage-typerelated AFLP fragment sequences. Of these, 14 sets were based on bacteriophage ST64B (Ross \& Heuzenroeder, 2005), 12 on bacteriophages P22, Gifsy-1 and Gifsy-2 (Mikasova et al., 2005), and six on the AFLP fragments (Hu et al., 2002). All primers were designed or modified so that they were $18-28 \mathrm{bp}$ in length, with melting temperatures between 59 and $71{ }^{\circ} \mathrm{C}$ and product sizes in the range 90-249 bp (Table 2). The nucleotide-nucleotide BLAST program (National Center for Biotechnology Information) was used to compare primer sequences with sequences in the GenBank database, to minimize cross-priming with other regions of phage genomes or unrelated genes. All primers were $5^{\prime}$ and $3^{\prime}$ biotin labelled and supplied by Sigma-Genosys.

Probes. Thirty-eight probes were designed, based on amplicon sequences of each primer set: two for each of the six primer sets based on AFLP fragment sequences and one for each of the other primer sets. All probes were designed with lengths between 18 and 27 bp and melting temperatures of $61-66{ }^{\circ} \mathrm{C}$ (Table 3$)$. BLAST searches of the
GenBank database were performed to minimize cross-hybridization with other genes. Probes were $5^{\prime}$ amino-labelled and supplied by Sigma-Genosys.

Multiplex PCR system. A $30 \mu$ multiplex PCR mixture containing 32 primer sets was prepared using the Qiagen Hotstar Taq PCR system as follows: $3.0 \mu \mathrm{l}$ of $10 \times$ PCR buffer, $4.5 \mu \mathrm{M} \mathrm{MgCl}_{2}, 0.2 \mathrm{mM}$ $\mathrm{dNTP}, 0.5 \mu \mathrm{M}$ each forward and reverse primer, $2 \mu \mathrm{l}$ template DNA,

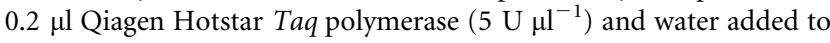
a total volume of $30 \mu \mathrm{l}$. A touchdown PCR was performed according to the protocol described previously (Ross \& Heuzenroeder, 2005) with decreasing primer-annealing temperatures from 59 to $52{ }^{\circ} \mathrm{C}$. One representative isolate from each of 46 phage types was also tested by single PCR using each primer set to validate the mPCR results.

RLB hybridization. The RLB hybridization assay was performed based on a well-established protocol developed in this laboratory (Kong \& Gilbert, 2006) with modifications. Probes were attached to a Biodyne Nylon 6.6 membrane (PALL; Gelman Laboratory). The hybridization temperature was $60{ }^{\circ} \mathrm{C}$. Streptavidin-peroxidase (Roche Diagnostics) was used as conjugate and diluted 1:4000 in $2 \times \operatorname{SSPE}\left(0.18 \mathrm{M} \mathrm{NaCl}, 10 \mathrm{mM} \mathrm{NaH} \mathrm{PO}_{4}\right.$ and $1 \mathrm{mM}$ EDTA, $\mathrm{pH}$ 7.7) containing $0.5 \%$ SDS. The X-ray film (Hyperfilm; Amersham) exposure time was $7 \mathrm{~min}$.

Sequencing. Sequencing was performed, using single PCR amplicons, to compare sequences of the corresponding targets from different isolates that gave strong or weak signals in RLB. The sequencing was conducted in both directions with BigDye Terminator version 3.7 (Applied Biosystems) on an ABI 3100 DNA sequencer.

MLVA. The MLVA typing method was used as described by Lindstedt et al. (2004) with the following modifications. A $30 \mu \mathrm{l}$ multiplex PCR mix was prepared using the Qiagen HotStar Taq PCR system containing $3.0 \mu \mathrm{l}$ of $10 \times$ PCR buffer, $2.5 \mathrm{mM} \mathrm{MgCl}_{2}, 0.2 \mathrm{mM} \mathrm{dNTP}$, $0.1 \mu \mathrm{M}$ each forward and reverse primer, $2 \mu \mathrm{l}$ template DNA, $0.2 \mu \mathrm{l}$ Hotstar Taq polymerase $\left(5 \mathrm{U} \mathrm{\mu l}^{-1}\right)$ and water added to a total volume of $30 \mu \mathrm{l}$. PCR products were run on a $1.6 \%$ normal agarose gel prior to the capillary electrophoresis. A single target PCR was run for any isolates with missing mPCR amplicons, to confirm absence of amplification. The capillary electrophoresis solution was prepared as follows: $0.5 \mu \mathrm{l}$ of the PCR products was mixed with $0.5 \mu$ lof the ROX1000 (Bio-Ventures) internal size marker and $9 \mu$ formamide. The mixed solution was then subjected to capillary electrophoresis on an ABI 3100 Genetic Analyzer (Applied Biosystems).

Data analysis. For each probe, RLB hybridization signal intensities on the membrane were scored as 2 for strong (gene present), 1 for weak (gene present with minor sequence variation) and 0 for absent (gene absent or present with major sequence variations) signals. These were combined into a 38-digit string for each isolate representing the RLB pattern. The string was divided into seven gene groups (G1-G7) each containing six gene markers, except for G7, which comprised only two. A single digit difference in the string was regarded as a different RLB type. The data were analysed using BioNumerics (Applied Maths) software and each unique pattern was given a number. A dendrogram was generated using the categorical coefficient and Ward's algorithm.

MLVA peaks were identified according to colours and sizes using GENESCAN software provided by Applied Biosystems and were binned into alleles. The allele numbers were entered into BioNumerics as character values. The number of repeats at each locus was calculated by subtracting the known length of the flanking sequence from the amplicon length and dividing the result by the repeat sequence length for all loci, except STTR3, which contains variable numbers of either 27 or $33 \mathrm{bp}$ repeats. The lengths of flanking sequences were 
determined from the $S$. Typhimurium LT2 complete genome sequence from GenBank (accession no. NC_003197). An allele string for each isolate, in the order STTR9-STTR5-STTR6-STTR10plSTTR3, was constructed by combining allele numbers, corresponding with the number of repeats ( 0 if there was no amplicon; 1 for flanking sequence only; 2 for one repeat; and 3 for two repeats and so on), for the first four loci. For STTR3, the allele numbers were based on eight amplicon size variants among all isolates tested ranging from $370 \mathrm{bp}$ (allele 1) to $523 \mathrm{bp}$ (allele 8). Different MLVA types were numbered consecutively as they were identified, based on differences in combinations of allele numbers.

Polymorphism indices were calculated using Simpson's index of diversity, as described by Hunter \& Gaston (1988).

\section{RESULTS}

\section{MPCR/RLB}

The 168 S. Typhimurium isolates were classified into 102 RLB patterns or types, which were numbered in order as shown in Fig. 1. The polymorphism index for RLB typing was 0.9866 . Fig. 2 shows a representative RLB profile of selected phage types of $S$. Typhimurium. Of the 168 isolates, 99 were clustered into 33 RLB types, each of which contained multiple isolates with identical patterns and, in most cases, corresponded with different phage types. Each of the remaining 69 isolates gave a unique RLB pattern. All RLB types could be further clustered into five groups (A-E; Fig. 1).

Of 33 RLB types with multiple isolates, one (RLB 92) contained isolates belonging to four phage types; two (RLB 41 and 95) contained three phage types; eleven (RLB 1, 45, $50,50,57,59,65,66,69,78,81$ and 87) contained two phage types; and 19 RLB types each contained a single phage type, some of which were shared between RLB types (Fig. 1).

All phage types with multiple isolates, except PT 99 and PT U290, produced two or more RLB patterns. For some phage types, the majority of isolates had the same RLB patterns. For example, four of five PT 101 isolates shared RLB 87, four of six PT 126 isolates belonged to RLB 3 and three of four untypable isolates shared RLB 61. Most other phage types showed significant differences in RLB patterns between isolates and some RLB patterns were shared between phage types. For example, six PT 1 isolates had five different RLB patterns spread among cluster groups B, C and E, namely RLB 25, 26, 33, 47 and 95 (the latter was shared with PT 5 and 136). Six PT 6 var1 isolates produced five RLB patterns spread between groups A, C and E, namely RLB 11, 42, 81, 82 and 83 (the latter was shared with PT 140 var1). Four PT 141 isolates each belonged to a different RLB type, namely RLB 18, 24, 92 and 94 (RLB 92 was shared with PT 4, 193 and 195). Similarly, each of five PT U302 isolates gave a different RLB pattern, namely RLB 12, 62, 64, 68 and 69 (RLB 69 was shared with PT 186) (Fig. 1).

Of the 46 phage types tested, 21 gave distinct RLB patterns, i.e. not shared by any other phage types, including 14 with multiple isolates (PT 12a, 20 var1, 30, 43, 44, 64, 104L, 126, 126 var4, 160, 197, 208, U290 and untypable) and seven with single isolates (PT 1 var, 4 var2, 29, 164, 165, 177 and 194). The number of distinct RLB patterns for each of these phage types varied between one and five. For two phage types, PT 126 var4 and PT 197, each of four and five isolates, respectively, gave different RLB patterns.

The numbers of gene target differences, between different RLB patterns for the same phage type, varied between 1 and 26 of the 38 probe targets. Of the isolates tested, PT 1 ( six isolates) and PT 195 (four isolates) were the most variable, with 26 and 23 differences, respectively. Among phage types with distinct RLB patterns, PT 208 and PT 197 had 12 and 10 target differences between isolates, respectively.

The probes CA4-P2, CC2-P2 and gtgB-P1 produced strong positive signals for all phage types and therefore could be used as control markers in future work. Amplicons which produced different signal intensities with the same probe were sequenced. The results showed that those producing strong signals (score 2) were identical to corresponding probe sequences; those that gave weak signals (scored as 1) had one (isolates 146, 158 and 187) or more than one (isolates 127 and 128) nucleotide base difference. Isolates that produced amplicons (as shown by single PCR and gel electrophoresis), but gave no signal with the corresponding probe (scored as 0 ), differed by more than two nucleotides (isolates 100, 123, 146, 158 and 187) (Table 4).

\section{MLVA typing}

The loci showed varied degrees of polymorphism from 21 alleles of STTR 5 to five of STTR9. The polymorphism indices for each locus were as follows: STTR3, 0.63; STTR5, 0.91; STTR6, 0.92; STTR9, 0.55; and STTR10pl, 0.84. The combination of five loci gave a polymorphism index of 0.9854. Three loci, STTR3, STTR5 and STTR9, were present in all isolates tested, while STTR6 and STTR10pl were absent from $6(3.6 \%)$ and $25(14.9 \%)$ isolates, respectively.

The 168 S. Typhimurium isolates were separated into 97 MLVA allele strings which were numbered from 1 to 97 (Fig. 1). Twenty-six of the 97 MLVA types were represented by multiple isolates, and most of these corresponded with a single phage type. For example, MLVA types 1, 6 and 25 corresponded with PT 160, PT 44 and PT U307, respectively, and MLVA 12 corresponded with PT 135 (six of 15 PT 135 isolates belonged to other MLVA types).

Some MLVA types contained two or more phage types, suggesting close relationships between them. For example, MLVA 2 contained five phage types, MLVA 84 contained four and MLVA 35 and 69 each contained two. On the other hand, even small numbers of some phage types were represented by several MLVA types; for example, six PT 1 isolates were represented by four MLVA types, 15 PT 135 isolates were represented by seven MLVA types and five PT 101 isolates were represented by five MLVA types (Fig. 1).

In many cases, individual RLB patterns included multiple MLVA types and vice versa; for example, RLB 41 contained 
Isolate $^{\dagger}$ RLB type MLVA type (pattern) Phage type

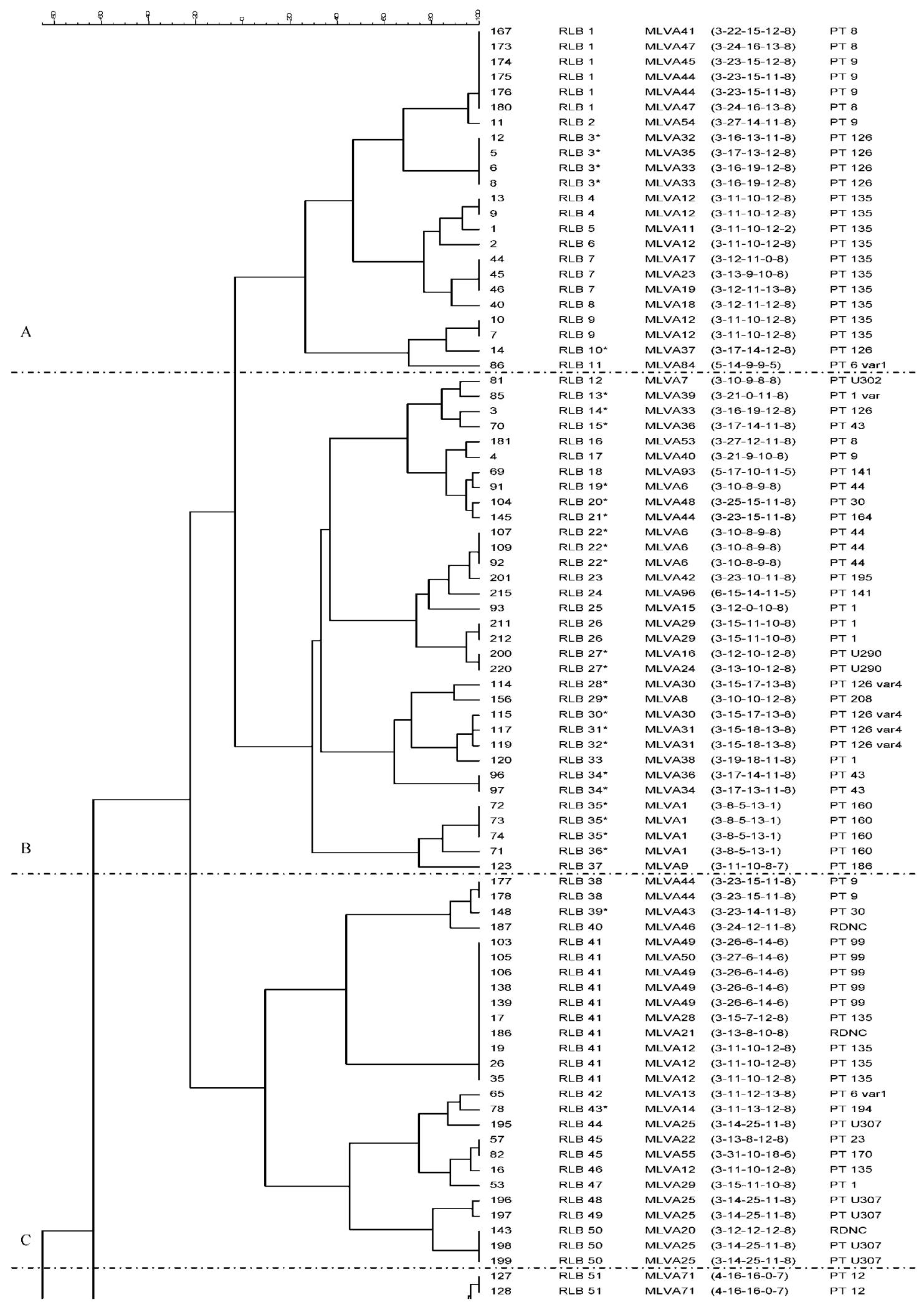




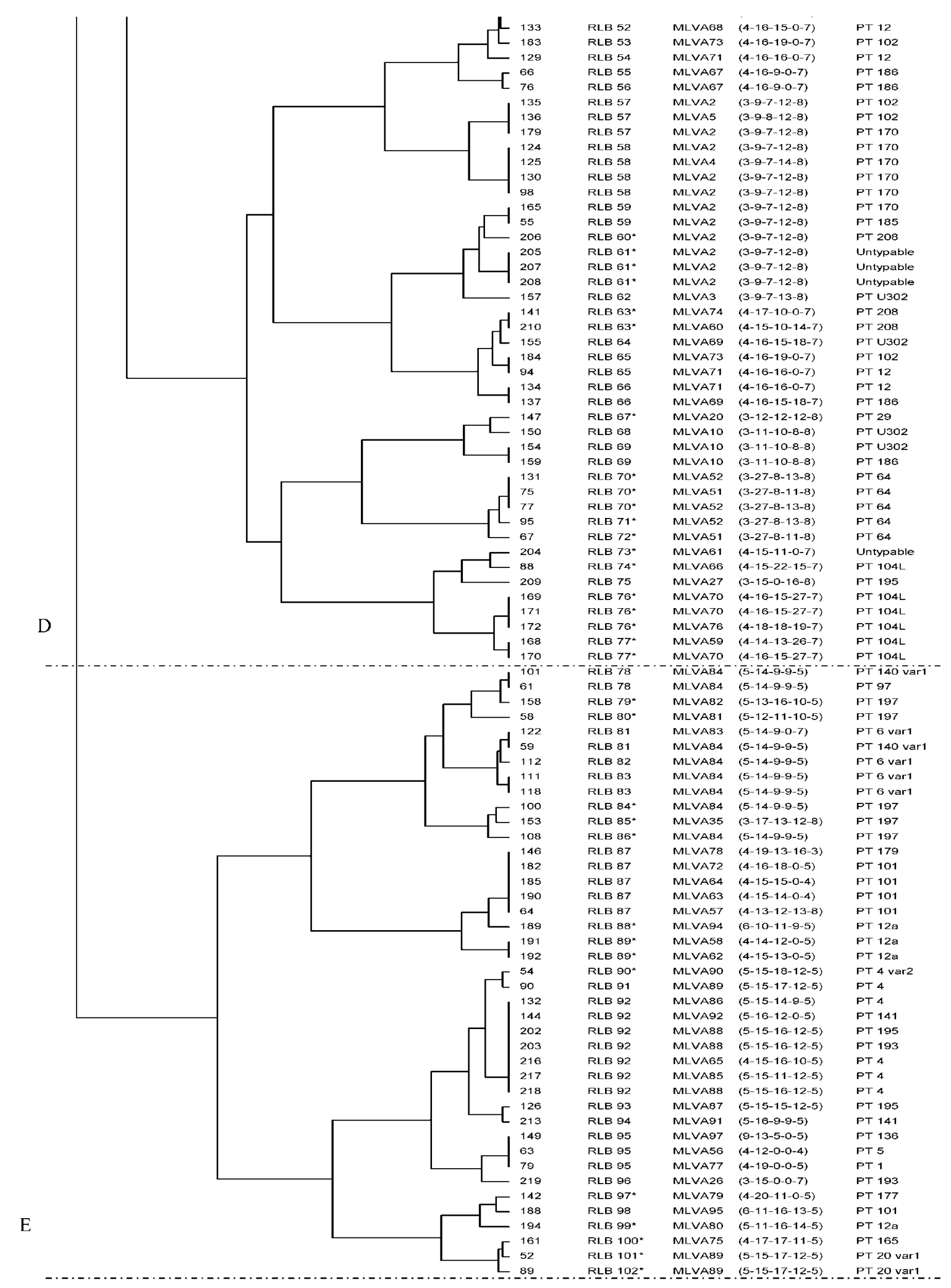

Fig. 1. Comparison of molecular typing methods using mPCR/RLB and MLVA for 168 S. Typhimurium isolates of 46 phage types. The dendrogram is divided into five larger groups, A, B, C, D and E, indicated by horizontal lines. tlsolates 17, 19, 26 and 35 (RLB 41) were from the drug and alcohol rehabilitation centre outbreak in New South Wales in 2004; *RLB types unique for specific phage types. 
Table 1. Isolates tested in the investigation of three outbreaks using mPCR/RLB and MLVA

\begin{tabular}{|c|c|c|c|c|c|}
\hline $\begin{array}{l}\text { Phage } \\
\text { type }\end{array}$ & $\begin{array}{l}\text { No. of isolates } \\
\text { tested }\end{array}$ & $\begin{array}{c}\text { RLB pattern } \\
(n)^{\star}\end{array}$ & $\begin{array}{c}\text { MLVA type } \\
(n) \dagger\end{array}$ & MLVA pattern $\ddagger$ & Source \\
\hline \multirow[t]{4}{*}{ PT 135} & 23 & RLB 41 (23) & $12(20)$ & $3-11-10-12-8$ & $\begin{array}{l}\text { Outbreak 1: drug and alcohol rehabilita- } \\
\text { tion centre outbreak in NSW (2004) }\end{array}$ \\
\hline & & & $98(1)$ & $3-10-10-10-8$ & \\
\hline & & & $99(1)$ & $3-5-15-1-8$ & \\
\hline & & & $28(1)$ & $3-15-7-12-8$ & \\
\hline \multirow[t]{8}{*}{ PT 135} & 15 & RLB 41 (11) & $16(5)$ & $3-12-10-12-8$ & Sporadic isolates (2001-2003) \\
\hline & & & $100(3)$ & $3-11-10-10-8$ & \\
\hline & & & $101(2)$ & $3-12-10-8-8$ & \\
\hline & & & $102(1)$ & $3-12-9-10-8$ & \\
\hline & & RLB 7 (4) & $103(1)$ & $3-12-11-10-8$ & \\
\hline & & & $8(1)$ & $3-10-10-12-8$ & \\
\hline & & & $12(1)$ & $3-11-10-12-8$ & \\
\hline & & & $23(1)$ & $3-13-9-10-8$ & \\
\hline \multirow[t]{2}{*}{ PT 126} & 2 & RLB 3 (2) & $33(1)$ & $3-16-19-12-8$ & \\
\hline & & & $37(1)$ & $3-17-14-12-8$ & \\
\hline \multirow[t]{3}{*}{ PT 170} & 14 & RLB 103 (14) & $5(12)$ & $3-9-8-12-8$ & $\begin{array}{l}\text { Outbreak 2: restaurant outbreaks in NSW } \\
\text { (2006) }\end{array}$ \\
\hline & & & $2(2)$ & $3-9-7-12-8$ & \\
\hline & 1 & RLB 103 (1) & $2(1)$ & $3-9-7-12-8$ & Food isolate \\
\hline \multirow[t]{9}{*}{ PT 170} & 22 & RLB 103 (17) & $5(12)$ & $3-9-8-12-8$ & Sporadic isolates (2000-2005) \\
\hline & & & $2(3)$ & $3-9-7-12-8$ & \\
\hline & & & $4(1)$ & $3-9-7-14-8$ & \\
\hline & & & $104(1)$ & $3-9-9-12-8$ & \\
\hline & & RLB 104 (1) & $55(1)$ & $3-31-10-18-6$ & \\
\hline & & RLB 105 (1) & $105(1)$ & $3-10-8-12-8$ & \\
\hline & & RLB 106 (2) & $106(1)$ & $4-15-9-0-7$ & \\
\hline & & & $107(1)$ & $4-16-8-0-7$ & \\
\hline & & RLB 107 (1) & $5(1)$ & $3-9-8-12-8$ & \\
\hline \multirow[t]{3}{*}{ PT 170} & 17 & RLB 58 (17) & $108(17)$ & $3-11-7-12-8$ & $\begin{array}{l}\text { Outbreak 3: catering college outbreak in } \\
\text { NSW (2006) }\end{array}$ \\
\hline & 2 & RLB 58 (2) & $108(2)$ & $3-11-7-12-8$ & Chocolate mousse \\
\hline & 2 & RLB 58 (2) & $2(2)$ & $3-9-7-12-8$ & PT 170 controls \\
\hline \multirow[t]{2}{*}{ PT 126} & 2 & RLB 3 (1) & $33(1)$ & $3-16-19-12-8$ & PT 126 controls \\
\hline & & RLB 10 (1) & $37(1)$ & $3-17-14-12-8$ & \\
\hline \multirow[t]{2}{*}{ PT 135} & 3 & RLB 4 (2) & $12(2)$ & $3-11-10-12-8$ & PT 135 controls \\
\hline & & RLB 8 (1) & $18(1)$ & $3-12-11-12-8$ & \\
\hline PT U290 & 2 & RLB 27 (2) & $16(2)$ & $3-12-10-12-8$ & PT U290 controls \\
\hline S. Java & 2 & RLB 108 (2) & S. Java 1 (2) & $1-13-0-0-4$ & Fish \\
\hline \multirow[t]{2}{*}{ S. Sofia } & 2 & RLB 109 (2) & S. Sofia 1 (1) & $0-10-0-0-0$ & Chicken \\
\hline & & & Not typed (1) & $0-0-0-0-0$ & Chicken \\
\hline
\end{tabular}

${ }^{\star}$ RLB types are numbered consecutively as they are identified, based on a 38-digit string determined by probe reaction strengths. RLB types 103-109 are new types not identified among 168 isolates initially tested.

$\dagger$ MLVA types are numbered consecutively as they are identified, based on a 5-digit string or pattern. MLVA types 98-108 are new types not identified among the 168 isolates initially tested.

¥MLVA pattern consists of a number of repeats $(+1)$ present at loci STTR9, STTR5, STTR6 and STTR10 (in that order) and allele number for STTR3 [see text for further details: $0=$ no amplicon; $1=$ amplicon equivalent to flanking region (no repeat sequence present)].

five MLVA types and MLVA 12 contained five RLB types (Fig. 1), indicating that RLB and MLVA types are generally complementary and together provide a higher level of discrimination than either alone. However, there were some exceptions, where the RLB, MLVA and PT types were consistent. For example, four PT 99 isolates shared the same RLB 41 and MLVA 49 types, three PT 44 isolates shared RLB 22 and MLVA 6 types, and three untypable isolates had the same RLB 61 and MLVA2 types (Fig. 1). Further information is required to determine whether isolates with consistent RLB, MLVA and phage types are epidemiologically related. 
Table 2. Primers used in the multiplex PCR for identifying phage types of Salmonella Typhimurium

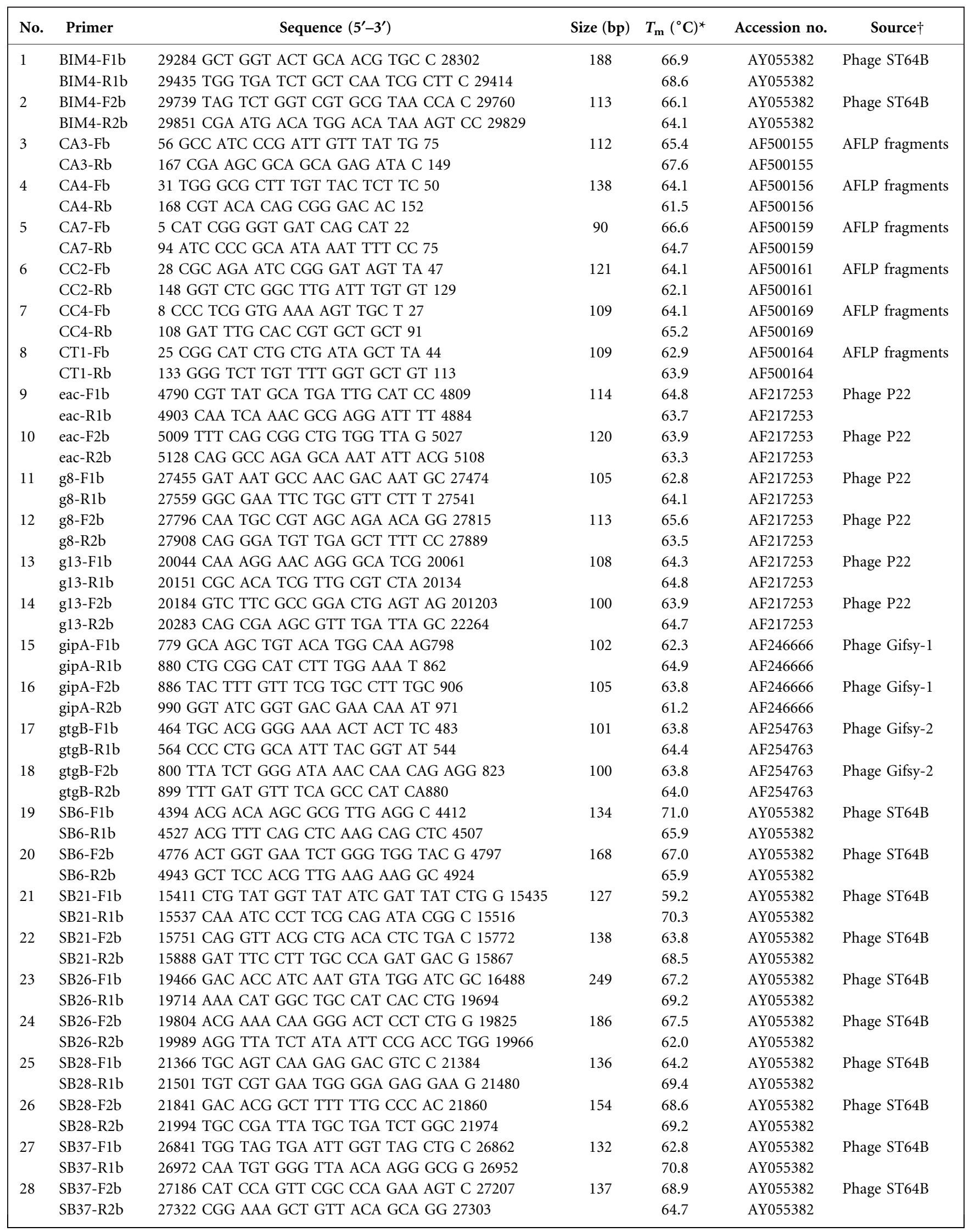


Table 2. cont.

\begin{tabular}{|c|c|c|c|c|c|c|}
\hline No. & Primer & Sequence $\left(5^{\prime}-3^{\prime}\right)$ & Size (bp) & $T_{\mathrm{m}}\left({ }^{\circ} \mathrm{C}\right)^{*}$ & Accession no. & Source $\dagger$ \\
\hline \multirow[t]{2}{*}{29} & SB46-F1b & 33640 CAT TGA TGG TAT CGA AGT TCG CC 33662 & 135 & 68.2 & AY055382 & Phage ST64B \\
\hline & SB46-R1b & 33774 TCA ATC AGC TCA CGG GTT TGC 33754 & & 69.4 & AY055382 & \\
\hline \multirow[t]{2}{*}{30} & SB46-F2b & 34001 AGT GCG CCA CAA ACA TCT TCT G 34022 & 130 & 68.4 & AY055382 & Phage ST64B \\
\hline & SB46-R2b & 34130 CCT GGA GTT TCT GGC ACG C 34112 & & 68.2 & AY055382 & \\
\hline \multirow[t]{2}{*}{31} & SieB-F1b & 11520 ATG GTG GCA GGA GTT AAT GC 11539 & 107 & 63.7 & AF217253 & Phage P22 \\
\hline & SieB-R1b & 11626 CGC TTA CCG GCA TAA CAA TAA 11606 & & 63.4 & AF217253 & \\
\hline \multirow[t]{2}{*}{32} & SieB-F2b & 11718 CGG TGT TGT TAA TTC CGT TT 11737 & 104 & 61.2 & AF217253 & Phage P22 \\
\hline & SieB-R2b & 11821 CAA ACA AAT CCC GAA CGA CT 11802 & & 63.7 & AF217253 & \\
\hline
\end{tabular}

*The melting temperature $\left(T_{\mathrm{m}}\right)$ was provided by the primer synthesizer (Sigma-Aldrich).

$\dagger$ All primers designed by modifications based on AFLP fragments refer to Hu et al. (2002), bacteriophage ST64B refers to Ross \& Heuzenroeder (2005) and bacteriophage P22, Gifsy-1 and -2 refer to Mikasova et al. (2005).

\section{Outbreak investigations}

Outbreak 1. The drug and alcohol rehabilitation centre outbreak in 2004 involved 23 residents from whom isolates were obtained. Nineteen residents had a diarrhoeal illness during one week in July; three other cases occurred in September and one isolate was collected from a chef at the centre in October. The outbreak was believed to be either food-borne or person-to-person spread. All 23 isolates belonged to PT 135 . The mPCR/RLB clearly distinguished PT 126 from PT 135 isolates. All of the latter had the same RLB type, which was the same as one of two RLB types observed among the sporadic isolates (Table 1). Twenty PT 135 outbreak isolates ( 16 from July, 3 from September and one from the chef in October) belonged to MLVA 12 and each of the other three isolates was a different type, as were the two PT 126 isolates. By contrast, there were eight MLVA types (including MLVA 12) among 15 sporadic PT 135 isolates (Table 1). Epidemiological investigation, before the molecular typing results were available, failed to identify a common source. The MLVA results suggest that there may have been more than one source but this could not be confirmed.

Outbreak 2. The epidemiological investigation of the restaurant outbreaks in 2006 identified two clusters and a possible, but unconfirmed, third. The first cluster involved three cases linked to a restaurant, which also provided a food isolate from prawns. The second cluster of three cases was related to a nearby café, which had the same chicken supplier as the restaurant. Two other affected individuals had eaten food from a pizza shop and another had eaten at the same pizza shop and the cafe implicated in the second cluster. No epidemiological links were identified for five other cases.

All 15 'outbreak' isolates produced the same RLB pattern (RLB 103), which was also the most common (represented by 17 of 22 isolates) of five patterns observed among the sporadic isolates. The 14 human isolates were divided into two MLVA types (MLVA 2 and 5), which differed by a single allele. The prawn isolate and two isolates from cluster 2 belonged to MLVA 2 and the remaining 12 belonged to MLVA 5, the most common of the seven MLVA types found among the sporadic isolates (Table 1).

Outbreak 3. This outbreak occurred over a short period among students attending a residential catering college, who cooked and ate together, which suggested a single common food source. All 17 human isolates from the students had the same RLB 58 type, which was the same as that of the PT 170 controls. DNA from four food isolates hybridized with only one to three of 38 RLB targets, suggesting that they were not $S$. Typhimurium. The other two, from chocolate mousse, belonged to the same RLB 58 type as the human isolates.

All human isolates and the two from chocolate mousse also had the same MLVA type (MLVA 107), which differed at only one locus from MLVA 2, which had previously been shown to be associated with PT 170 . The other four food isolates did not produce amplicons from two to five of the loci.

Outbreak isolates from the affected students and the chocolate mousse were later identified as $S$. Typhimurium PT 170. The other food isolates were identified as two Salmonella Sofia isolates, from chicken, and two Salmonella Java isolates, from fish. Epidemiological investigation implicated chocolate mousse, which contained raw egg, as the most likely common food source (Table 1).

\section{DISCUSSION}

Our aim was to develop a molecular typing system for $S$. Typhimurium that could, potentially, replace or supplement phage typing, which has significant limitations and limited discriminatory ability. Improvements in commercial 'hot start' PCR Taq polymerase technology have made it easier to obtain all expected amplification products from increasing numbers of primers in a single multiplex reaction. If mPCR is used in combination with RLB hybridization, each 
Table 3. Probes designed for the RLB hybridization

\begin{tabular}{|c|c|c|c|c|}
\hline No. & Probe $^{\star}$ & Sequence $\left(5^{\prime}-3^{\prime}\right)$ & $T_{\mathrm{m}}\left({ }^{\circ} \mathrm{C}\right) \dagger$ & Accession no \\
\hline 1 & BIM4-P1 & 28305 AGG AAC CTA TCA CCT GGC AC 28324 & 62.9 & AY055382 \\
\hline 2 & BIM4-P2 & 29809 CGG TTA AGT TCC TGC ACG 29792 & 64.5 & AY055382 \\
\hline 4 & CA3-P2 & 148 CGA TCA GGA AAG TAT CCA CTG G 127 & 64.5 & AF500155 \\
\hline 5 & CA4-P1 & 52 GAC CAT CTT ACC TAC CTG TTC TGG 75 & 63.6 & AF500156 \\
\hline 8 & CA7-P2 & 71 AGA CAT TTT TAC CGG ACC GG 52 & 60.8 & AF500159 \\
\hline 9 & CC2-P1 & 49 CAA TCC AAA ACA GAC CGA CAG 69 & 65.9 & AF500161 \\
\hline 10 & $\mathrm{CC} 2-\mathrm{P} 2$ & 125 CAG TTA TTT GGT TGT TCC CTG 105 & 60.8 & AF500161 \\
\hline 11 & CC4-P1 & 31 CAG CTC ACG GGT TTG CTT AT 50 & 64.0 & AF500169 \\
\hline 12 & CC4-P2 & 72 CGT CCG AAA TAC TGG CTT GAT A 51 & 64.8 & AF500169 \\
\hline 13 & CT1-P1 & 49 TCG ATA ATG CCA ACG ACA AT 68 & 62.8 & AF500164 \\
\hline 17 & g8-P1 & 27481 GGA AGA GGG CTT TGA GAT TGT C 27502 & 64.9 & AF217253 \\
\hline 18 & g8-P2 & 27863 CTT CGA CGT ATT GCG CTG A 27845 & 65.1 & AF217253 \\
\hline 19 & g13-P1 & 20068 TCC TTG CGT TTG CAA TGG 20085 & 65.8 & AF217253 \\
\hline 20 & g13-P2 & 20249 GTG CCG ATG TAG CCG ATA 20231 & 65.2 & AF217253 \\
\hline 21 & gipA-P1 & 804 AAT CCC CTT AGA CGT GCG 821 & 62.6 & AF246666 \\
\hline 22 & gipA-P2 & 963 ACC AAC ATC AAT GCC GAC C 945 & 65.8 & AF246666 \\
\hline 23 & gtgB-P1 & 492 GGA TGA AGG CAG TCA GGA GAT ATT 515 & 65.3 & AF254763 \\
\hline 24 & gtgB-P2 & 879 CAC ATA GTC TTT TCC TTC TTT ATT TCC 853 & 62.0 & AF254763 \\
\hline 25 & SB6-P1 & 4421 AGG AAA AGG GCA AGC TTG 4440 & 61.9 & AY055382 \\
\hline 26 & SB6-P2 & 4890 CCG TAG ATT TCC CCC ATG A 4871 & 64.5 & AY055382 \\
\hline 27 & SB21-P1 & 15439 GGA ATA TGC TGC CTG ATA TGT G 15460 & 63.0 & AY055382 \\
\hline 28 & SB21-P2 & 15842 GGT ATC CAG TAA TAG CGT TCC A 15821 & 61.6 & AY055382 \\
\hline 36 & SB46-P2 & 34110 CC CAG TAC CGA TGA ATT GGA 34091 & 64.5 & AY055382 \\
\hline 37 & SieB-P1 & 11547 CCT GCA AGG AAT GAC ACT TAA AC 11569 & 63.4 & AF217253 \\
\hline 38 & SieB-P2 & 11800 TTT CTT CTC TGT CCT TAC GCC 11780 & 62.2 & AF217253 \\
\hline
\end{tabular}

*Probes were designed on both strands inside primer sites listed in Table 3.

$\dagger$ The melting temperature $\left(T_{\mathrm{m}}\right)$ was provided by the primer synthesizer (Sigma-Aldrich).

individual amplification product can be identified by its reaction with a specific probe on a nylon membrane array.

Based on published sequence data, we designed or modified 32 primer sets targeting sequences from phages ST64B (Ross \& Heuzenroeder, 2005), P22, Gifsy-1 and Gifsy-2 (Mikasova et al., 2005) and S. Typhimurium phage type-related AFLP fragments (Hu et al., 2002). They were designed to produce short PCR products with similar physical and biochemical characteristics to allow simultaneous amplification in a multiplex reaction. Similarly, probes were designed so that their optimal hybridization conditions were similar. We designed two primer sets and probes for phage-related targets in order to increase the number of nucleotide polymorphisms identified and therefore the discriminatory ability of the assay. Each member of the pair serves as an internal control for the other. Because of their short length (100-150 bp), only single primer pairs and probes were used for targets based on phage type-related AFLP fragments.

This system identified and discriminated subtypes among many of the phage types tested. The RLB patterns were defined by the presence or absence and intensities of hybridization signals; differences in intensity between paired probes were confirmed by sequencing results. The mPCR results were validated by performing single PCRs for each primer set on one representative of each of the 46 


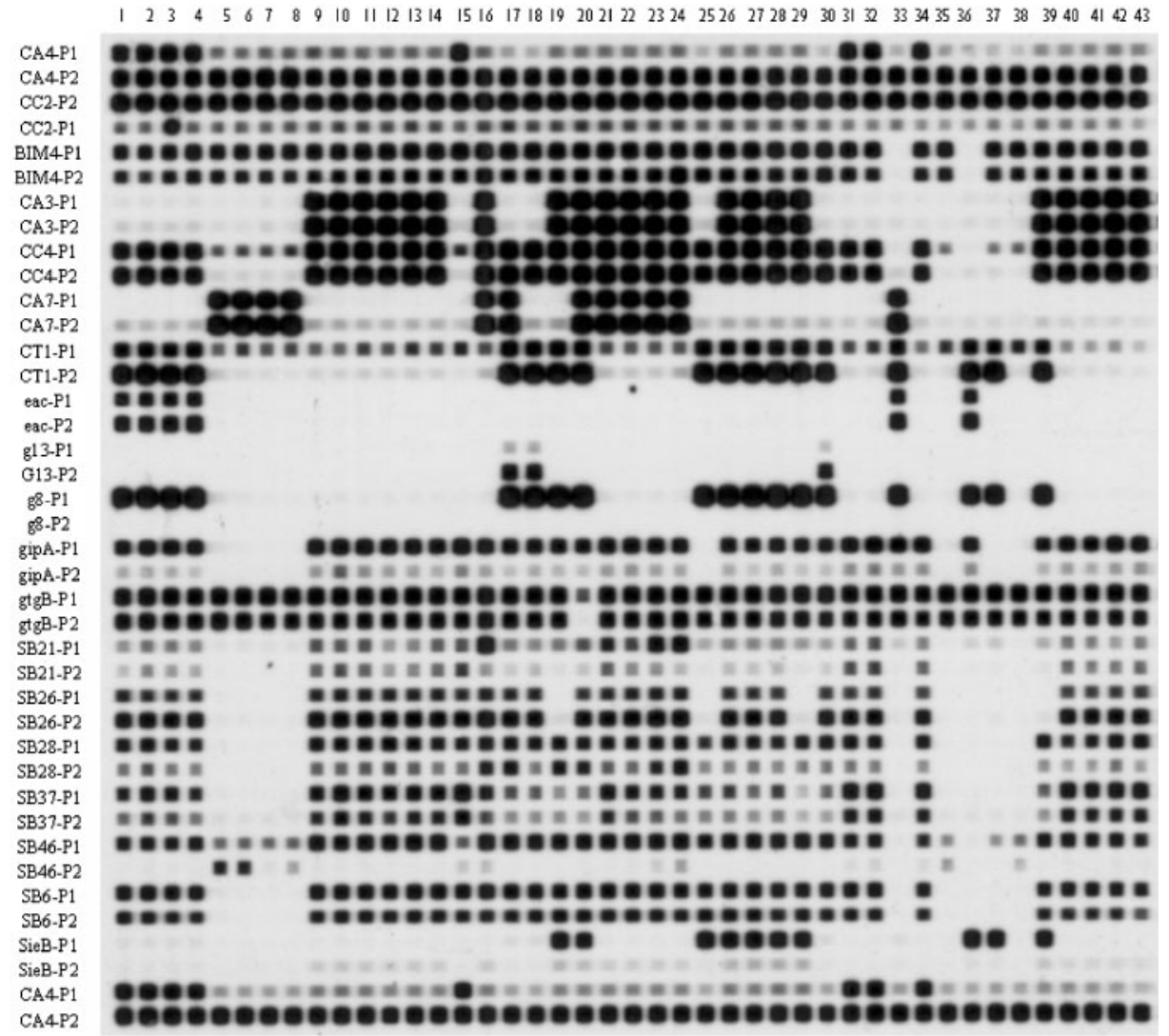

Fig. 2. RLB patterns of isolates of $S$. Typhimurium from selected phage types. Lanes: 1-4 (PT 64), isolates 75, 77 (RLB 70 ), 95 (RLB 71), 131 (RLB 70); 5-8 (PT 6 var1), isolates 118, 111 (RLB 83), 112 (RLB 82), 122 (RLB 81); 9-13 (PT 99), isolates 103, 105, 106, 138, 139 (RLB 41); 14-16 (RDNC), isolates 186 (RLB 41), 187 (RLB 40), 143 (RLB 50); 17-20 (PT U302), isolates 150 (RLB 68), 154 (RLB 69), 155 (RLB 64), 157 (RLB 62); 21-24 (PT U307), isolates 196 (RLB 48), 197 (RLB 49), 198, 199 (RLB 50); 25-28 (untypable), isolates 204 (RLB 73), 205, 207, 208 (RLB 61); 29-30 (PT 186), isolates 137 (RLB 66), 159 (RLB 69); 31-32 (PT 9), isolates 177, 178 (RLB 38); 33 (PT 20 var1), isolate 89 (RLB 102); 34 (PT 30), isolate 148 (RLB 39); 35 (PT 140 var1), isolate 101 (RLB 78); 36 (PT 177), isolate 142 (RLB 97); 37 (PT 179), isolate 146 (RLB 87); 38 (PT 197), isolate 158 (RLB 79); 39 (PT 12), isolate 134 (RLB 66); 40-43 (PT 135), isolates 17, 19, 26, 35 (RLB 41). Left column: probe list.

phage types studied. The method reliably differentiated 21 of 46 individual phage types including some that are closely related, such as PT12 and PT12a. Some common phage types shared RLB types with one other, e.g. PT 8 with 9, PT 12 and 102 with 170, and PT 99 and RDNC with 135, suggesting a close relationship between members of the pair or group. Isolates of most of the common phage types exhibited two or more RLB patterns, indicating the potential for more discriminatory strain typing of common phage types. Multiple RLB patterns were found among the untypable and RDNC isolates, indicating that they represented diverse groups of largely unrelated strains.

The application of this novel mPCR/RLB system to the investigation of outbreaks confirmed its potential value. The first outbreak was suspected when phage-typing results demonstrated an increased number of cases due to the common phage type 135 within a short period of time. The combination of mPCR/RLB and MLVA helped to define the clusters and identify related cases some time after they had occurred. In the second outbreak, all 15 isolates from three apparently related restaurant outbreaks had the same RLB pattern, which was also one of five patterns exhibited by sporadic isolates of S. Typhimurium PT 170. These results support epidemiological data and are compatible with the supposition that the three outbreaks were related to each other and to the food sample. In the third outbreak, investigation began immediately after salmonellae were isolated from cases and three types of food. The outbreak strain and the food source were rapidly identified by $\mathrm{mPCR} / \mathrm{RLB}$ and predicted the phage type involved more than a week before the phage typing results were available.

Had it been performed routinely on all $S$. Typhimurium isolates, the mPCR/RLB typing alone would have provided 
Table 4. Sequencing results based on scoring of RLB hybridization signals

\begin{tabular}{|c|c|c|c|c|}
\hline Isolate & Signal $^{\star}$ & $\begin{array}{l}\text { Sequence } \dagger \text { (probe CA4-P1: } \\
\text { CCAGAACAGGTAGGTAAGATGGTC) }\end{array}$ & Signal $^{\star}$ & $\begin{array}{l}\text { Sequence } \dagger \text { (probe CA4-P2: } \\
\text { GTGCGTAATTTGCGTTGTTT) }\end{array}$ \\
\hline 174 & 2 & CCAGAACAGGTAGGTAAGATGGTC & 2 & GTGCGTAATTTGCGTTGTTT \\
\hline 127 & 1 & CCAGAACA - - - GGTAAGATGGTC & 2 & GTGCGTAATTTGCGTTGTTT \\
\hline 128 & 1 & CCAGAACA - - - GGTAAGATGGTC & 2 & GTGCGTAATTTGCGTTGTTT \\
\hline 100 & 0 & CCAGAACA - - - GGTAAGATGGTC & 2 & GTGCGTAATTTGCGTTGTTT \\
\hline \multirow{2}{*}{123} & & Sequence (probe CC4-P1: & & Sequence (probe CC4-P2: \\
\hline & & ATAAGCAAACCCGTGAGCTG) & & TATCAAGCCAGTATTTCGGAC) \\
\hline 17 & 2 & ATAAGCAAACCCGTGAGCTG & 2 & TATCAAGCCAGTATTTCGGAC \\
\hline 134 & 2 & ATAAGCAAACCCGTGAGCTG & 2 & TATCAAGCCAGTATTTCGGAC \\
\hline 143 & 2 & ATAAGCAAACCCGTGAGCTG & 2 & TATCAAGCCAGTATTTCGGAC \\
\hline 146 & 1 & ATAAGCAAACCCGTGAACTG & 0 & TATCAAGCCAGTACTTGGGAC \\
\hline
\end{tabular}

${ }^{\star}$ The signal scoring was as described in Methods.

$\nmid$ The deletion of a nucleotide is indicated by '-' and the nucleotide sequence different from the probe is underlined.

early warning of the first two outbreaks, before the phage typing or epidemiological data were available. For the third outbreak, which was immediately apparent because of the large number of related cases presenting within a short period, it rapidly defined the outbreak strain and confirmed the food source. It is more sensitive and specific than phage typing and results are available much sooner.

MLVA is also highly sensitive for strain typing, especially in outbreaks. Three of the loci tested, STTR5, STTR6 and STTR10pl, displayed a high degree of polymorphism indicated by indices of $0.91,0.92$ and 0.84 , respectively, which were comparable with published work (Lindstedt et al., 2004). One of the major drawbacks of this typing strategy is that the generation of variation in some MLVA loci is under selection pressure such that two identical clones may appear to differ if isolates have originated from different niches or from hosts with altered immune responses (Lindstedt, 2005). This may be manifested as too high a level of discrimination to correctly cluster isolates from a common source in an outbreak investigation. The PT 135 isolates from the first outbreak were divided into four MLVA types, of which one was predominant and the others differed by two to three alleles. It is not clear whether the three isolates belonging to minor MLVA types were sporadic or variants of the outbreak strain. Further experience with both RLB and MLVA is required to assist interpretation of these results.

Both mPCR/RLB assay and MLVA demonstrated similar discriminatory power in this study. For some phage types, the strains identified were the same between the two methods. For example, both methods separated PT 30, PT 141 and RDNC into different molecular types for each isolate tested (Fig. 1). Some phage types contained the same number of molecular types identified by both methods; for example, four isolates of PT 102 contained three molecular types and seven isolates of PT 9 contained four. Both methods demonstrated a high level of similarity between isolates of some groups of phage types such as PT 135, PT 102 and PT 170 (Fig. 1).

Based on these results, mPCR/RLB is as sensitive and specific as MLVA. Both methods can provide informative strain typing data and can presumptively identify many common phage types within $24 \mathrm{~h}$, compared with at least 3 weeks for phage typing at a reference laboratory. This difference represents a highly significant advantage for prompt identification of an outbreak source. An advantage of mPCR/RLB over MLVA is that the PCR amplicons are detected by hybridization rather than fragment size, which overcomes the problem of reproducibility between different platforms. Based on our results, mPCR/RLB showed less variation than MLVA among outbreak isolates, which made interpretation easier. MLVA is less labour intensive than mPCR/RLB and apparently more sensitive for outbreak investigations, but further experience with both methods is required to determine which more accurately reflects the epidemiology.

mPCR/RLB is a promising tool that can be developed further by the introduction of additional gene markers, including serotype-specific markers, to increase its sensitivity and discriminatory ability for Salmonella species other than $S$. Typhimurium. It has the potential to replace phage typing in the future.

\section{ACKNOWLEDGEMENTS}

This work was supported by a National Health and Medical Research Council project grant (\#457472). We thank Ms Jennie Musto from NSW Department of Health for providing epidemiological informa- 
tion and Dr Michael Heuzenroder and Ian Ross from the Australian Salmonella Reference Centre, Adelaide, for providing assistance in primer selection. Thanks to Vitali Sintchenko for proof reading of the manuscript. Thanks also to Robert Chiew and Peter Howard for assisting in the selection of isolates from the Enteric Reference Laboratory in CIDM.

\section{REFERENCES}

Anderson, E. S., Ward, L. R., Saxe, M. J. \& de Sa, J. D. (1977). Bacteriophage-typing designations of Salmonella Typhimurium. J Hyg (Lond) 78, 297-300.

Cho, S., Boxrud, D. J., Bartkus, J. M., Whittam, T. S. \& Saeed, M. (2007). Multiple-locus variable-number tandem repeat analysis of Salmonella enteritidis isolates from human and non-human sources using a single multiplex PCR. FEMS Microbiol Lett 275, 16-23.

Davos, D. (editor) (2006). Most Common Phage Types from Major Sources, p. 10. Annual Report, Australian Salmonella Reference Centre, Institute of Medical and Veterinary Science, Adelaide, Australia.

De Cesare, A., Manfreda, G., Dambaugh, T. R., Guerzoni, M. E. \& Franchini, A. (2001). Automated ribotyping and random amplified polymorphic DNA analysis for molecular typing of Salmonella enteritidis and Salmonella Typhimurium strains isolated in Italy. J Appl Microbiol 91, 780-785.

Fakhr, M. K., Nolan, L. K. \& Logue, C. M. (2005). Multilocus sequence typing lacks the discriminatory ability of pulsed-field gel electrophoresis for typing Salmonella enterica serovar Typhimurium. J Clin Microbiol 43, 2215-2219.

Guerra, B., Schrors, P. \& Mendoza, M. C. (2000). Application of PFGE performed with $\mathrm{XbaI}$ to an epidemiological and phylogenetic study of Salmonella serotype Typhimurium. Relations between genetic types and phage types. New Microbiol 23, 11-20.

Hu, H., Lan, R. \& Reeves, P. R. (2002). Fluorescent amplified fragment length polymorphism analysis of Salmonella enterica serovar Typhimurium reveals phage-type- specific markers and potential for microarray typing. J Clin Microbiol 40, 3406-3415.

Hunter, P. R. \& Gaston, M. A. (1988). Numerical index of the discriminatory ability of typing systems: an application of Simpson's index of diversity. J Clin Microbiol 26, 2465-2466.

Jeoffreys, N. J., James, G. S., Chiew, R. \& Gilbert, G. L. (2001) Practical evaluation of molecular subtyping and phage typing in outbreaks of infection due to Salmonella enterica serotype Typhimurium. Pathology 33, 66-72.

Kong, F. \& Gilbert, G. L. (2006). Multiplex PCR-based reverse line blot hybridization assay (mPCR/RLB) - a practical epidemiological and diagnostic tool. Nat Protoc 1, 2668-2680.

Lan, R., Stevenson, G., Donohoe, K., Ward, L. \& Reeves, P. R. (2007). Molecular markers with potential to replace phage typing for
Salmonella enterica serovar Typhimurium. J Microbiol Methods 68, $145-156$

Lindstedt, B. A. (2005). Multiple-locus variable number tandem repeats analysis for genetic fingerprinting of pathogenic bacteria. Electrophoresis 26, 2567-2582.

Lindstedt, B. A., Heir, E., Vardund, T. \& Kapperud, G. (2000). A variation of the amplified-fragment length polymorphism (AFLP) technique using three restriction endonucleases, and assessment of the enzyme combination BglII-MfeI for AFLP analysis of Salmonella enterica subsp. enterica isolates. FEMS Microbiol Lett 189, 19-24.

Lindstedt, B. A., Heir, E., Gjernes, E. \& Kapperud, G. (2003). DNA fingerprinting of Salmonella enterica subsp. enterica serovar Typhimurium with emphasis on phage type DT104 based on variable number of tandem repeat loci. J Clin Microbiol 41, 1469-1479.

Lindstedt, B. A., Vardund, T., Aas, L. \& Kapperud, G. (2004). Multiple-locus variable-number tandem-repeats analysis of Salmonella enterica subsp. enterica serovar Typhimurium using PCR multiplexing and multicolor capillary electrophoresis. J Microbiol Methods 59, 163-172.

Liu, Y., Lee, M. A., Ooi, E. E., Mavis, Y., Tan, A. L. \& Quek, H. H. (2003). Molecular typing of Salmonella enterica serovar Typhi isolates from various countries in Asia by a multiplex PCR assay on variablenumber tandem repeats. J Clin Microbiol 41, 4388-4394.

Mikasova, E., Drahovska, H., Szemes, T., Kuchta, T., Karpiskova, R., Sasik, M. \& Turna, J. (2005). Characterization of Salmonella enterica serovar Typhimurium strains of veterinary origin by molecular typing methods. Vet Microbiol 109, 113-120.

Millemann, Y., Lesage, M. C., Chaslus-Dancla, E. \& Lafont, J. P. (1995). Value of plasmid profiling, ribotyping, and detection of IS200 for tracing avian isolates of Salmonella Typhimurium and $S$. enteritidis. J Clin Microbiol 33, 173-179.

Nastasi, A. \& Mammina, C. (1995). Epidemiological evaluation by PCR ribotyping of sporadic and outbreak-associated strains of Salmonella enterica serotype Typhimurium. Res Microbiol 146, 99106.

Popoff, M. Y., Bockemuhl, J. \& Gheesling, L. L. (2004). Supplement 2002 (no. 46) to the Kauffmann-White scheme. Res Microbiol 155, 568-570.

Ross, I. L. \& Heuzenroeder, M. W. (2005). Discrimination within phenotypically closely related definitive types of Salmonella enterica serovar Typhimurium by the multiple amplification of phage locus typing technique. J Clin Microbiol 43, 1604-1611.

Soria, G., Barbe, J. \& Gibert, I. (1994). Molecular fingerprinting of Salmonella Typhimurium by IS200-typing as a tool for epidemiological and evolutionary studies. Microbiologia 10, 57-68.

Witonski, D., Stefanova, R., Ranganathan, A., Schutze, G. E., Eisenach, K. D. \& Cave, M. D. (2006). Variable-number tandem repeats that are useful in genotyping isolates of Salmonella enterica subsp. enterica serovars Typhimurium and Newport. J Clin Microbiol 44, 3849-3854. 